Flávia NeVes Bueloni-Dias ${ }^{1}$

Daniel Spadoto-Dias ${ }^{1}$

JORGE NAHÁS-NETO

Eliana Aguiar Petri Nahás 1

\title{
Fatores preditivos para a ocorrência de pólipos endometriais em mulheres na pós-menopausa
}

\author{
Predictive factors for occurrence of \\ endometrial polyps in postmenopausal women
}

Artigo Original

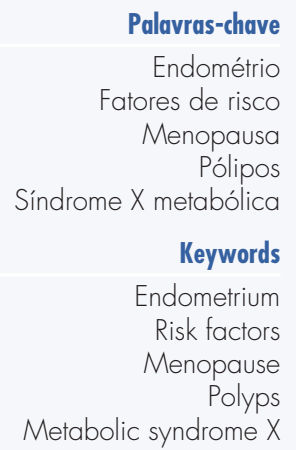

Correspondência

Flávia Neves Bueloni-Dias

Departamento de Ginecologia e Obstetrícia Faculdade de Medicina de Botucatu, Universidade Estadual Paulista

"Júlio de Mesquita Filho"

Distrito de Rubião Júnior, $s$ /

CEP: 18618-970

Botucatu (SP), Brasil

Recebido

15/07/2014

Aceito com modificacões

12/09/2014

\section{Resumo}

OBJETIVO: Avaliar os fatores clínicos preditivos para o desenvolvimento dos pólipos endometriais em mulheres na pós-menopausa. MÉTODOS: Estudo de coorte observacional com mulheres na pós-menopausa, que haviam sido atendidas em hospital público universitário. Dados clínicos, antropométricos, laboratoriais e ultrassonográficos de 132 pacientes com diagnóstico anatomopatológico de pólipo endometrial e de 264 mulheres sem alterações endometriais (controle) foram comparados para avaliar os fatores preditivos do pólipo endometrial. Foram incluídas no estudo mulheres com amenorreia $\geq 12$ meses e idade $\geq 45$ anos, em uma proporção de 1 caso para 2 controles. Para a análise estatística, foram empregados os testes $t$ de Student, $\chi^{2}$ e regressão logística - odds ratio (OR). RESULTADOS: As pacientes com pólipo endometrial apresentaram idade mais avançada e maior tempo de menopausa quando comparadas ao controle ( $p<0,0001)$. A porcentagem de mulheres obesas com pólipo $(72,0 \%)$ foi superior à do Grupo Controle (39\%; p<0,000 1). A medida da circunferência da cintura foi superior entre as pacientes com pólipo ( $p=0,0001$ ). Observou-se uma incidência de diabetes, hipertensão e dislipidemias mais elevada nas pacientes com pólipo endometrial ( $<<0,0001)$. De acordo com os critérios do US National Cholesterol Education Program/ Adult Treatment Panel III (NCEP/ATP III), 48,5\% das mulheres com pólipo e 33,3\% do Grupo Controle foram classificadas como portadoras de síndrome metabólica $(p=0,004)$. Em relação ao Grupo Controle, apresentaram maior chance de desenvolvimento de pólipo endometrial as pacientes com: IMC $\geq 25 \mathrm{~kg} / \mathrm{m}^{2} 1 \mathrm{OR}=4,6$; IC95\% 2, 1-10,0); glicose $\geq 100 \mathrm{mg} / \mathrm{dL} \mid \mathrm{OR}=2,8$; IC95\% 1,3-5,9); dislipidemia $(O R=7,0$; IC95\% 3,7-13,3); diabetes $|\mathrm{OR}=2,5 ;| \mathrm{IC} 95 \%$ 1,0-6,3) e síndrome metabólica $|\mathrm{OR}=2,7 ;| \mathrm{C} 95 \% 1,1-6,4)$. CONCLUSÃO: Em mulheres na pós-menopausa, obesidade, dislipidemia, hiperglicemia e presença de síndrome metabólica foram fatores preditivos para o desenvolvimento de pólipo endometrial.

\section{Abstract}

PURPOSE: To evaluate the predictive clinical factors for the development of endometrial polyps in postmenopausal women. METHODS: Observational cohort study with postmenopausal women who had been at a public university hospital. Clinical, anthropometrical, laboratorial, and ultrasonographic data of 132 patients with a histopathological diagnosis of endometrial polyps and 264 women without endometrial alterations (control) were compared in order to evaluate the predictive factors of endometrial polyps. Women with amenorrhea $\geq 12$ months and $\geq 45$ years of age were included in the study at a proportion of 1 case for 2 controls. The Student's $F, \chi^{2}$, and logistic regression tests were used for statistical analysis - odds ratio (OR). RESULTS: Patients with endometrial polyps were older and had been in menopause for a longer time compared to control ( $<<0.0001)$. The percentage of obese women with polyps (72.0\%) was higher compared to the Control Group (39\%; $<<0.0001$ ). The measurement of waist circumference was superior among patients with polyps
Setor de Climatério e Menopausa do Departamento de Ginecologia e Obstetrícia da Universidade Estadual Paulista "Júlio de Mesquita Filho" - UNESP - Botucatu (SP), Brasil.

'Departamento de Ginecologia e Obstetrícia, Faculdade de Medicina de Botucatu, Universidade Estadual Paulista "Júlio de Mesquita Filho" - UNESP - Botucatu (SP), Brasil.

Conflito de interesses: não há 
$(p=0.0001)$. We observed a higher incidence of diabetes, hypertension and dyslipidemia in patients with endometrial polyps ( $<<0.0001)$. According to the US National Cholesterol Education Program/Adult Treatment Panel III (NCEP/ATP III) criteria, 48.5\% of women with polyps and 33.3\% of the Control Group were classified as having metabolic syndrome $(\mathrm{p}=0.004)$. Analysis of risk for endometrial polyps formation showed higher chances of occurrence of the disorder in patients with: $\mathrm{BMl} \geq 25 \mathrm{~kg} / \mathrm{m}^{2}(\mathrm{OR}=4.6 ; 95 \% \mathrm{Cl} 2.1-10.0)$; glucose $\geq 100 \mathrm{mg} / \mathrm{dL}(\mathrm{OR}=2.8 ; 95 \% \mathrm{Cl}$ 1.3-5.9); dyslipidemia (OR=7.0; 95\%Cl 3.7-13.3); diabetes $\mid O R=2.5 ; 95 \% \mathrm{Cl}$ 1.0-6.3), and metabolic syndrome $(O R=2.7 ; 95 \% \mathrm{Cl} 1.1-6.4)$ compared to the Control Group. CONCLUSION: In postmenopausal women, obesity, dyslipidemia, hyperglycemia and presence of metabolic syndrome were predictive factors for the development of endometrial polyps.

\section{Introdução}

Pólipos endometriais são neoformações focais da camada basal do endométrio que acometem entre 7,8 e $34,9 \%$ das mulheres, a depender do método diagnóstico utilizado e da população estudada ${ }^{1-3}$. Sua prevalência é maior entre mulheres que se encontram na faixa etária dos 40 aos 60 anos, e duas vezes maior entre aquelas na pós-menopausa, comparativamente ao período de menacme ${ }^{2,4}$. São responsáveis por 21 a $28 \%$ de todas as causas de sangramento uterino na pós-menopausa, configurando relevante condição para o diagnóstico diferencial da neoplasia endometrial ${ }^{5,6}$.

A incidência do câncer de endométrio associado aos pólipos está em torno de 3,5\%, variando segundo a maioria dos estudos entre 0,0 e $4,8 \%$, sendo que esse risco é maior em mulheres na pós-menopausa com sangramento ${ }^{6,7}$. Como fatores de risco para o desenvolvimento de pólipos endometriais, bem como para degeneração maligna dos mesmos, são considerados idade avançada, nuliparidade, menarca precoce, menopausa tardia, obesidade, diabetes, hipertensão e uso de tamoxifeno ${ }^{8,9}$.

A síndrome metabólica (SM) é definida como um conjunto de fatores de riscos metabólicos que incluem obesidade abdominal, dislipidemia, hipertensão arterial e disglicemia (resistência à insulina, intolerância à glicose ou diabetes $)^{10,11}$. Resistência à insulina e distúrbios do metabolismo de carboidratos são comuns em mulheres com doenças endometriais ${ }^{12}$. Estudos epidemiológicos têm relatado associações de risco de desenvolvimento do câncer endometrial com os componentes individuais da $\mathrm{SM}$, com destaque para obesidade, hiperglicemia, hipertensão arterial e aumento nos níveis de triglicérides, o que poderia também associar a SM aos pólipos endometriais; porém, esse envolvimento permanece incerto ${ }^{13-15}$.

A prevalência dos pólipos endometriais na pós-menopausa é bastante significativa, sendo causa comum de sangramento uterino anormal, o que ocasiona recorrentes discussões quanto à sua diferenciação com a neoplasia do endométrio. Em virtude da ampla utilização da ultrassonografia transvaginal (USTV) na avaliação ginecológica de rotina, o achado incidental de espessamentos difusos ou focais da camada endometrial sugere, com frequência, uma investigação mais invasiva, com a realização de histeroscopia diagnóstica e biópsia endometrial, uma vez que nenhum parâmetro ultrassonográfico mostrou-se fidedigno na diferenciação entre pólipo e câncer endometrial ${ }^{7}$. Diante da observação de um aumento na prevalência dos pólipos endometriais concomitantemente à obesidade e SM, o presente estudo procurou avaliar fatores clínicos preditivos do desenvolvimento dos pólipos endometriais em mulheres na pós-menopausa.

\section{Métodos}

\section{Desenho do estudo e seleção da amostra}

Tratou-se de um estudo observacional de coorte, analítico e comparativo. O grupo populacional foi constituído de pacientes atendidas nos Ambulatórios de Histeroscopia e Climatério \& Menopausa, da Faculdade de Medicina de Botucatu (FMB), São Paulo, entre 2000 e 2013. O cálculo do tamanho amostral foi embasado no estudo de Miranda et al. ${ }^{16}$, os quais relatam a ocorrência de pólipo endometrial em aproximadamente 30,0\% das mulheres na pós-menopausa. Considerando-se essa frequência, com nível de significância de 5\%, margem de erro de $8 \%$ e IC95\%, estimou-se a necessidade de avaliar, no mínimo, 126 mulheres com pólipo endometrial.

Foram incluídas na pesquisa mulheres com data da última menstruação há pelo menos 12 meses e idades $\geq 45$ anos. No grupo de estudo, as pacientes apresentavam diagnóstico anatomopatológico de pólipo endometrial após a realização de polipectomia. Os laudos eram fornecidos pelo Departamento de Patologia da FMB (Figura 1). A partir de um banco de dados, o Grupo Controle foi constituído de mulheres sem história de sangramento na pós-menopausa e sem espessamento endometrial à USTV, com eco endometrial $\leq 5 \mathrm{~mm}$, na proporção de 1 caso para 2 controles (1:2). Não participaram da análise pacientes com dados clínicos incompletos, sendo incluídas no estudo 132 mulheres com pólipo endometrial e 264 controles assintomáticos na pós-menopausa.

Foram esclarecidos, para as participantes, os objetivos do estudo e os procedimentos aos quais seriam submetidas, sendo solicitadas suas assinaturas do Termo de Consentimento Livre e Esclarecido (TCLE), exigência da Resolução no 196, de outubro de 1996 do Conselho Nacional de Saúde do Ministério da Saúde, após aprovação pelo Comitê de Ética e Pesquisa da FMB da Universidade Estadual Paulista "Júlio de Mesquita Filho" (UNESP) (Protocolo 4201-2012). 


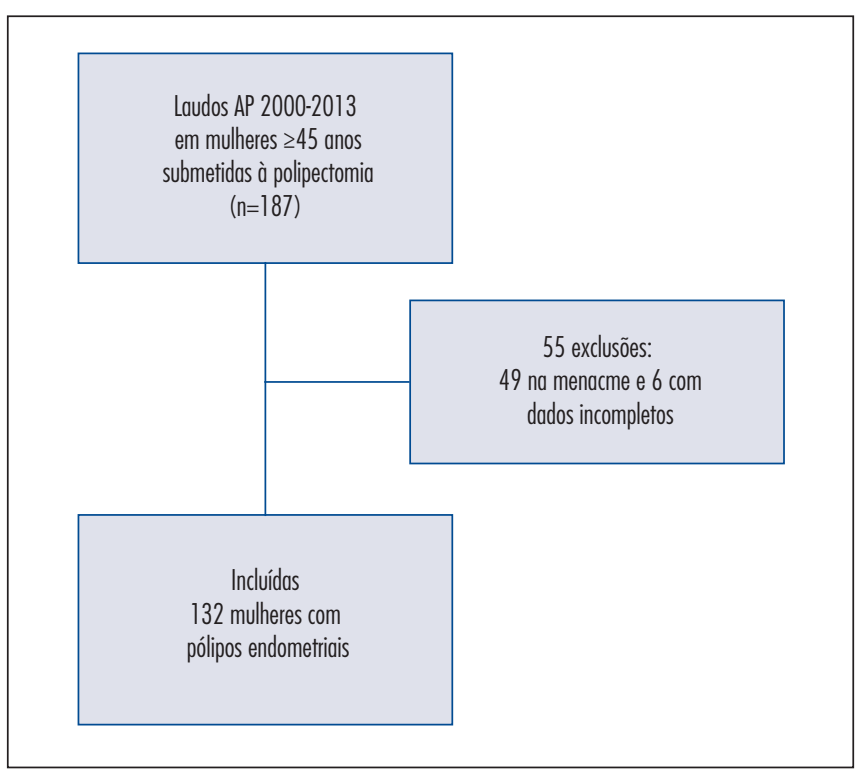

Figura 1. Fluxograma das pacientes com pólipo endometrial incluídas no estudo.

\section{Avaliações clínica, laboratorial e ultrassonográfica}

A partir de análise do prontuário médico das pacientes, foram coletadas as seguintes informações: idade, idade e tempo de menopausa, paridade, tabagismo, uso de terapia hormonal (TH), história pessoal de doença cardiovascular (DCV), hipertensão arterial sistêmica (HAS), diabetes, dislipidemias e pressão arterial. A medida da pressão arterial foi aferida com a paciente sentada, com o antebraço direito apoiado no nível do precórdio, palma da mão para cima, utilizando-se de esfigmomanômetro aneroide padrão. Foram consideradas com SM as mulheres que apresentaram três ou mais critérios diagnósticos propostos pelo US National Cholesterol Education Program/Adult Treatment Panel III (NCEP-ATP III), em $2001^{17}$; circunferência da cintura (CC) $>88 \mathrm{~cm}$; triglicerídeos $(\mathrm{TG}) \geq 150 \mathrm{mg} / \mathrm{dL}$; HDL colesterol $<50 \mathrm{mg} / \mathrm{dL}$; pressão arterial $\geq 130 / 85 \mathrm{mmHg}$; glicose de jejum $\geq 100 \mathrm{mg} / \mathrm{dL}$ ou sob terapia para qualquer uma dessas condições.

Para a avaliação antropométrica, foram coletados dados sobre: peso, altura, índice de massa corpórea $\left(\mathrm{IMC}=\right.$ peso/altura $\left.{ }^{2}\right)$ e circunferência de cintura (CC). Para mensuração do peso, utilizou-se balança antropométrica eletrônica, tipo plataforma da marca Filizola ${ }^{\circledR}$, graduada a cada $100 \mathrm{~g}$, com capacidade de até $150 \mathrm{~kg}$, precisão de $0,1 \mathrm{~kg}$, com a paciente descalça e vestindo pouca roupa. A estatura foi determinada em estadiômetro vertical afixado à balança, com precisão de 0,1 $\mathrm{cm}$, sendo a paciente orientada a manter-se em posição ortostática, com os braços ao lado do corpo, a cabeça orientada à frente, descalça, mantendo os pés juntos e em inspiração profunda. Foram empregados os critérios da World Health Organization de 2002 para classificação das pacientes conforme o IMC: $\leq 24,9 \mathrm{~kg} / \mathrm{m}^{2}$, normal; de 25 a $29,9 \mathrm{~kg} / \mathrm{m}^{2}$, sobrepeso; e $\geq 30,0 \mathrm{~kg} / \mathrm{m}^{2}$, obesidade. Para a medida da cintura, considerou-se a menor circunferência entre a última costela e a crista ilíaca anterossuperior, sendo a leitura realizada no momento da expiração e considerada aumentada quando acima de $88 \mathrm{~cm}^{17}$.

Foram realizadas avaliações dos perfis lipídico e glicídico pela mensuração do colesterol total (CT), HDL, LDL, TG e glicose de jejum, assim como da proteína C-reativa (PCR). Todas as avaliações bioquímicas foram aplicadas pelo Laboratório de Análises Clínicas da FMB. As mensurações de TG, CT, HDL, glicose e PCR foram processadas pelo analisador bioquímico automático, modelo Vitros $950^{\circledR}$, pelo método colorimétrico de química seca (Johnson \& Johnson, Rochester, NY, EUA). O método é linear até $800 \mathrm{mg} / \mathrm{dL}$ para TG e $900 \mathrm{mg} / \mathrm{dL}$ para CT. O LDL foi obtido pela fórmula de Friedewald et al. ${ }^{18}$, subtraindo-se o valor do CT, da soma do HDL e do TG dividido por cinco. Aqueles considerados ótimos foram: $\mathrm{CT}<200 \mathrm{mg} / \mathrm{dL}, \mathrm{HDL}>50 \mathrm{mg} / \mathrm{dL}$, $\mathrm{LDL}<100 \mathrm{mg} / \mathrm{dL}, \mathrm{TG}<150 \mathrm{mg} / \mathrm{dL}$, glicemia $<100 \mathrm{mg} / \mathrm{dL}$ e PCR $<1,0 \mathrm{mg} / \mathrm{dL}^{17,19}$.

$A$ avaliação da espessura endometrial foi realizada por meio da USTV pelo Serviço de Radiodiagnóstico da FMB. O aparelho Power Vision 6000 (Toshiba, Tóquio, Japão) foi aplicado com transdutor endovaginal de 7,5 $\mathrm{MHz}$. A espessura endometrial, medida na incidência sagital de uma camada basal à outra, foi considerada normal quando $\leq 5 \mathrm{~mm}^{20}$.

\section{Análise estatística}

Foram construídas as tabelas das variáveis que poderiam modificar o risco para o desenvolvimento de pólipo endometrial, comparando-se o grupo de mulheres na pós-menopausa submetidas à polipectomia e aquele sem ou outras alterações endometriais (controle). Para a análise foram calculados as médias e o desvio padrão para as variáveis quantitativas e os percentuais para as qualitativas. A fim de se comparar os grupos em relação às características quantitativas (clínicas, antropométricas e bioquímicas), empregou-se o teste $t$ de Student e a distribuição gama (para os dados com distribuição assimétrica). Para verificação da associação entre a frequência das características clínicas categóricas, utilizou-se o teste do $\chi^{2}$ ou exato de Fisher. Foi realizada análise multivariada por regressão logística binária, considerando-se o nível de significância $\mathrm{p}<0,05$ e IC95\%, com cálculo da respectiva odds ratio (OR). Neste caso, o grupo de mulheres foi submetido à polipectomia como resposta comparado ao controle como base, para se observarem as possíveis associações existentes entre o risco de desenvolvimento do pólipo endometrial (variável dependente) e as variáveis influentes do risco (variáveis independentes), sendo ajustada para idade e 
tempo de menopausa (variáveis confundidoras). Todas foram testadas por meio do ajuste do modelo de regressão logística múltipla, utilizando o procedimento "stepwise" para as variáveis que apresentassem diferença significativa. Os testes estatísticos foram bilaterais e o nível de significância adotado foi de $5 \%$. As análises foram realizadas utilizando-se o Statistical Analyses System (SAS), versão 9.2, pelo Escritório de Apoio à Pesquisa (EAP) da FMB, que forneceu o atendimento metodológico e conduziu os procedimentos estatísticos.

\section{Resultados}

As características clínicas, antropométricas, bioquímicas e ultrassonográficas das pacientes na pós-menopausa submetidas à polipectomia $(n=132)$ e ao controle $(n=264)$ estão representadas nas Tabelas 1 a 3 . Na Tabela 1 verifica-se que os grupos foram homogêneos para as seguintes variáveis: pressões arteriais sistólica e diastólica, valores de TG e PCR ( $p>0,05)$. Observou-se diferença significante quanto à idade, à idade e ao tempo de menopausa, à paridade, ao IMC, à CC e para os valores médios de CT, HDL, LDL, glicose e espessura endometrial.

As pacientes com pólipo endometrial apresentaram idade mais avançada (média de 60,7 versus 54,8 anos; $\mathrm{p}<0,0001$ ), maior média de idade de ocorrência da menopausa (média de 49,4 versus 47,6 anos; $\mathrm{p}=0,0001$ ) e maior tempo de menopausa (média de 11,3 versus 7,2 anos; $\mathrm{p}<0,0001)$ quando comparadas ao controle (Tabela 1 ). No grupo do pólipo endometrial, as mulheres tiveram mais filhos (média de 3,8 versus 2,8 filhos; $\mathrm{p}<0,0001$ ) do que no Grupo Controle. Os valores médios do IMC demonstraram que as pacientes com pólipo endometrial foram classificadas predominantemente como obesas, enquanto aquelas do Grupo Controle como sobrepeso $(\mathrm{p}<0,0001)$. A medida da CC foi superior entre as pacientes com pólipo endometrial (média de 115,6 versus $93,1 \mathrm{~cm} ; \mathrm{p}=0,0001)$ quando comparadas ao controle (Tabela 1). Os valores médios de CT $(\mathrm{p}=0,01)$, LDL $(\mathrm{p}=0,04)$ e glicose $(\mathrm{p}<0,0001)$ mostraram-se superiores nas mulheres com pólipo endometrial, enquanto o HDL mostrou-se inferior $(\mathrm{p}=0,01)$. A espessura endometrial encontrava-se em média quatro vezes maior nas pacientes com pólipo endometrial quando comparada ao Grupo Controle (média de 12,1 versus 3,1 mm; $\mathrm{p}<0,0001$ ), como visto na Tabela 1.

$\mathrm{Na}$ Tabela 2 observa-se a associação das características clínicas entre as mulheres na pós-menopausa, com e sem pólipo endometrial. Uma porcentagem superior de mulheres com pólipo endometrial encontrava-se na faixa etária entre 55 e 65 anos $(63,8 \%)$, enquanto que, no Controle, as mulheres encontravam-se em sua maioria abaixo dos 55 anos $(54,6 \%$; p<0,0001). Em relação ao
Tabela 1. Comparação das características clínicas, laboratoriais e ultrassonográficas entre as mulheres na pós-menopausa, com ( $\mathrm{n}=132$ ) e sem ( $\mathrm{n}=264$, controle) pólipo endometrial

\begin{tabular}{|c|c|c|c|}
\hline Parâmetros & $\begin{array}{c}\text { Pólipo } \\
\text { endometrial }\end{array}$ & Controle & Valor p \\
\hline Idade (anos), n (\%) & $60,7(6,4)$ & $54,8(6,6)$ & $<0,0001^{*}$ \\
\hline Idade da menopausa (anos), n (\%) & $49,4(4,4)$ & $47,6(4,2)$ & $0,0001^{*}$ \\
\hline Tempo de menopausa (anos), n (\%) & $11,3(7,6)$ & $7,2(5,8)$ & $<0,0001^{*}$ \\
\hline Paridade (número de filhos) ${ }^{\dagger}$ & $3,8 \pm 2,3$ & $2,8 \pm 1,8$ & $<0,0001^{*}$ \\
\hline IMC $\left(\mathbf{k g} / \mathrm{m}^{2}\right)^{\dagger}$ & $33,0 \pm 6,0$ & $29,0 \pm 5,2$ & $<0,0001^{*}$ \\
\hline $\mathrm{CC}(\mathrm{cm})^{\dagger}$ & $115,6 \pm 12,0$ & $93,1 \pm 12,9$ & $0,0001^{*}$ \\
\hline PAS $(m m H g)^{\dagger}$ & $130,6 \pm 15,3$ & $128,5 \pm 17,5$ & $0,2^{*}$ \\
\hline PAD $(m m H g){ }^{\dagger}$ & $81,3 \pm 10,2$ & $79,4 \pm 10,9$ & $0,1^{*}$ \\
\hline Colesterol total $(\mathrm{mg} / \mathrm{dL})^{\dagger}$ & $216,9 \pm 54,5$ & $203,5 \pm 35,1$ & $0,01^{*}$ \\
\hline$H D L(m g / d L)^{\dagger}$ & $49,4 \pm 12,7$ & $53,4 \pm 11,5$ & $0,01^{*}$ \\
\hline LDL $(\mathrm{mg} / \mathrm{dL})^{\dagger}$ & $130,8 \pm 40,5$ & $119,7 \pm 33,7$ & $0,04^{*}$ \\
\hline Triglicérides (mg/dL) ${ }^{\dagger}$ & $168,2 \pm 84,5$ & $152,2 \pm 66,6$ & $0,08^{\#}$ \\
\hline Glicose $(\mathrm{mg} / \mathrm{dL})^{\dagger}$ & $113,7 \pm 46,3$ & $94,6 \pm 20,7$ & $<0,0001^{*}$ \\
\hline PCR (mg/dL) ${ }^{\dagger}$ & $0,7 \pm 0,6$ & $0,6 \pm 0,5$ & $0,1^{\#}$ \\
\hline USTV EE (mm) ${ }^{\dagger}$ & $12,1 \pm 6,0$ & $3,1 \pm 1,2$ & $<0,0001^{*}$ \\
\hline
\end{tabular}

IMC: índice de massa corpórea: CC: circunferência da cintura: PAS: pressão arterial sistólica; PAD: pressão arterial diastólica; HDL: lipoproteína de alta densidade; LDL: lipoproteína de baixa densidade; PCR: proteína C-reativa; USTV EE: eco endometrial à ultrassonografia transvaginal. Diferença significativa $-p<0,05$;

*teste $t$ de Student e "teste de distribuição gama.

tempo de menopausa, verificou-se que $46,2 \%$ das pacientes com pólipo endometrial apresentavam mais de 10 anos de pós-menopausa, enquanto $48,9 \%$ das pacientes do Controle tinham menos de 5 anos $(\mathrm{p}<0,0001)$. Em ambos os grupos, a maioria das mulheres tinha mais de 2 filhos (66,7\% nos casos e 54,6\% nos controles), como observado na Tabela 2.

Entre as pacientes com pólipo endometrial, uma menor porcentagem relatou tabagismo atual, quando comparadas ao Grupo Controle (11,4 versus 20,5\%, respectivamente), com $\mathrm{p}=0,02$. Quanto aos antecedentes pessoais, observa-se maior incidência de diabetes, hipertensão e dislipidemias nas pacientes com pólipo endometrial $(28,8 ; 75,8$ e $56,1 \%$; respectivamente) quando comparadas ao controle $(6,8 ; 40,9$ e $13,3 \%$; respectivamente), com $\mathrm{p}<0,0001$. De acordo com os critérios NCEP/ATP III, $48,5 \%$ das mulheres com pólipo endometrial e 33,3\% do Grupo Controle foram classificadas como portadoras da SM, com diferença significativa entre os grupos ( $\mathrm{p}=0,004$; Tabela 2).

$\mathrm{Na}$ Tabela 3 verifica-se a associação das características laboratoriais e ultrassonográficas entre os grupos. Observa-se diferença significativa na distribuição percentual quanto ao HDL $(\mathrm{p}=0,02)$ e à glicose $(\mathrm{p}<0,0001)$, sendo que uma proporção menor de mulheres com pólipo endometrial tinha valores considerados ótimos quando comparadas ao Controle ( 47,7 versus $62,5 \%$ e 47,7 versus $79,2 \%$; respectivamente). O espessamento endometrial foi encontrado em $92,4 \%$ das pacientes com pólipo do endométrio e em nenhuma paciente do controle, conforme o critério de inclusão do grupo ( $<<0,0001$; Tabela 3). 
Tabela 2. Associacão das características clínicas entre as mulheres na pós-menopausa, com ( $n=132$ ) e sem ( $n=264$, controle) pólipo endometrial

\begin{tabular}{|c|c|c|c|}
\hline Características & $\begin{array}{c}\text { Pólipo } \\
\text { endometrial } \\
\text { n (\%) }\end{array}$ & $\begin{array}{l}\text { Controle } \\
\text { n (\%) }\end{array}$ & Valor $p^{*}$ \\
\hline Faixa etária (anos) & & & $<0,0001$ \\
\hline$<55$ & $20(15,2)$ & $144(54,6)$ & \\
\hline $55-65$ & $84(63,8)$ & $99(37,5)$ & \\
\hline$\geq 65$ & $28(21,0)$ & $21(7,9)$ & \\
\hline Tempo de menopausa (anos) & & & $<0,0001$ \\
\hline$\leq 5$ & $40(30,3)$ & $129(48,9)$ & \\
\hline $6-10$ & $31(23,5)$ & $75(28,4)$ & \\
\hline$>10$ & $61(46,2)$ & $60(22,7)$ & \\
\hline Paridade & & & 0,01 \\
\hline 0 & $5(3,8)$ & $31(11,7)$ & \\
\hline$\leq 2$ & $39(29,5)$ & $89(33,7)$ & \\
\hline$>2$ & $88(66,7)$ & $144(54,6)$ & \\
\hline Tabagismo atual & & & 0,02 \\
\hline $\operatorname{Sim}$ & $15(11,4)$ & $54(20,5)$ & \\
\hline Não & $117(88,6)$ & $210(79,5)$ & \\
\hline Uso de TH & & & 0,2 \\
\hline $\operatorname{Sim}$ & $22(16,7)$ & $58(22,0)$ & \\
\hline Não & $110(83,3)$ & $206(78,0)$ & \\
\hline IMC $\left(\mathrm{kg} / \mathrm{m}^{2}\right)$ & & & $<0,0001$ \\
\hline$\leq 24,9$ & $14(10,6)$ & $57(21,6)$ & \\
\hline $25,0-29,9$ & $23(17,4)$ & $104(39,4)$ & \\
\hline$\geq \mathbf{3 0}$ & $95(72,0)$ & $103(39,0)$ & \\
\hline Diabetes & & & $<0,0001$ \\
\hline Sim & $38(28,8)$ & $18(6,8)$ & \\
\hline Não & $94(71,2)$ & $246(93,2)$ & \\
\hline HAC & & & $<0,0001$ \\
\hline Sim & $100(75,8)$ & $108(40,9)$ & \\
\hline Não & $32(24,2)$ & $156(59,1)$ & \\
\hline Dislipidemia & & & $<0,0001$ \\
\hline Sim & $74(56,1)$ & $35(13,3)$ & \\
\hline Não & $58(43,9)$ & $229(86,7)$ & \\
\hline SM & & & 0,004 \\
\hline Sim & $64(48,5)$ & $88(33,3)$ & \\
\hline Não & $68(51,5)$ & $176(66,7)$ & \\
\hline
\end{tabular}

TH: terapia hormonal; IMC: índice de massa corpórea; HAC: hipertensão arterial crônica; SM: síndrome metabólica. * diferença significativa entre grupos $\left(p<0,05\right.$, teste do $\left.\chi^{2}\right)$

A análise de riscos para a formação de pólipo endometrial na presença de variáveis influentes, ajustados para idade e tempo de menopausa, demonstra maior chance de desenvolvimento do pólipo endometrial nas pacientes que apresentam: IMC $\geq 25 \mathrm{~kg} / \mathrm{m}^{2}(\mathrm{OR}=4,6$; IC95\% 2,1-10,0); pelo menos um filho $(\mathrm{OR}=5,1$; IC95\% 1,2-21,1); glicose $\geq 100 \mathrm{mg} / \mathrm{dL}(\mathrm{OR}=2,8$; IC95\% 1,3-5,9); dislipidemia (OR=7,0; IC95\% 3,7-13,3); diabetes $(\mathrm{OR}=2,5$; IC95\% 1,0-6,3) e SM $(\mathrm{OR}=2,7$; IC95\% 1,1-6,4). As demais variáveis analisadas não influenciaram significativamente o risco para o desenvolvimento do pólipo endometrial.
Tabela 3. Associacão das características laboratoriais e ultrassonográficas entre as mulheres na pós-menopausa, com ( $n=132$ ) e sem ( $n=264$, controle) pólipo endometrial

\begin{tabular}{|c|c|c|c|}
\hline Características & $\begin{array}{c}\text { Pólipo } \\
\text { endometrial } \\
\text { n (\%) }\end{array}$ & $\begin{array}{c}\text { Controle } \\
\text { n (\%) }\end{array}$ & Valor $\mathrm{p}^{*}$ \\
\hline Colesterol total (mg/dL) & & & 0,2 \\
\hline$<200$ & $59(44,7)$ & $137(51,9)$ & \\
\hline$\geq 200$ & $73(55,3)$ & $127(48,1)$ & \\
\hline$H D L(m g / d L)$ & & & 0,02 \\
\hline$\geq 50$ & $63(47,7)$ & $165(62,5)$ & \\
\hline$<50$ & $69(52,3)$ & $99(37,5)$ & \\
\hline LDL (mg/dL) & & & 0,5 \\
\hline$<100$ & $29(21,9)$ & $69(26,1)$ & \\
\hline$\geq 100$ & $103(78,1)$ & $195(73,9)$ & \\
\hline Triglicérides (mg/dL) & & & 0,4 \\
\hline$<150$ & $67(50,7)$ & $147(55,7)$ & \\
\hline$\geq 150$ & $65(49,3)$ & $117(44,3)$ & \\
\hline Glicose (mg/dL) & & & $<0,0001$ \\
\hline$<100$ & $63(47,7)$ & $209(79,2)$ & \\
\hline$\geq 100$ & $69(52,3)$ & $55(20,8)$ & \\
\hline USTV EE (mm) - média $\pm D P$ & & & $<0,0001^{\star \star}$ \\
\hline$\leq 5$ & $10 \pm 7,6$ & $264 \pm 100,0$ & \\
\hline$>5$ & $122 \pm 92,4$ & $0 \pm 0,0$ & \\
\hline
\end{tabular}

\section{Discussão}

Os pólipos endometriais são, atualmente, a principal indicação de histeroscopia ambulatorial e cirúrgica. Contudo, não há consenso para o manejo dessas lesões, bem como critérios ou marcadores bioquímicos que indiquem com segurança seu potencial de malignidade ${ }^{21}$. A observação da mudança dos padrões de esteroidogênese durante a menopausa, predispondo ao aparecimento dos pólipos endometriais e da obesidade central, associada à elevada ocorrência da SM, despertou o interesse por investigar marcadores clínicos que pudessem predizer o risco para o desenvolvimento dos pólipos nesse período específico da vida de uma mulher. No presente estudo, em mulheres na pós-menopausa, obesidade, dislipidemia, hiperglicemia e presença de SM foram fatores preditivos para o desenvolvimento de pólipo endometrial.

A SM está associada com modificações orgânicas sistêmicas, em especial a obesidade central que contribui para a resistência insulínica (RI), a dislipidemia e a hipertensão arterial, induzindo um estado pro-inflamatório com liberação de inúmeros mediadores, tais como PCR, fator de necrose tumoral, interleucina 6 e leptina ${ }^{22,23}$. No ambiente endometrial, a presença de alterações inflamatórias acompanha o padrão sistêmico, com liberação dos mesmos mediadores, podendo representar condição indispensável para o desenvolvimento de doenças 
endometriais $^{24}$. $\mathrm{Na}$ presente pesquisa, empregando-se os critérios NCEP/ATP III ${ }^{17}, 48,5 \%$ das mulheres com pólipo endometrial e 33,3\% do Grupo Controle foram classificadas como portadoras da SM, obtendo-se diferença significativa entre os grupos. Esses dados estão em concordância com vários estudos, nos quais se observou prevalência de SM em mulheres na pós-menopausa em porcentagens variando de 31 a $54,6 \%{ }^{25}$. $\mathrm{Na}$ análise do risco para pólipos endometriais, apresentaram maiores chances de desenvolvimento do pólipo as pacientes com IMC $\geq 25 \mathrm{~kg} / \mathrm{m}^{2}$, glicose $\geq 100 \mathrm{mg} / \mathrm{dL}$ e que apresentavam dislipidemia, diabetes e SM. Estudos epidemiológicos têm relatado associações de risco para o desenvolvimento do câncer endometrial com os componentes individuais da SM, incluindo obesidade, diabetes e hipertensão, com possível associação também com os pólipos endometriais ${ }^{13-15}$. Contudo, outras investigações têm demonstrado que, embora hipertensão, diabetes e obesidade sejam variáveis significativamente associadas aos pólipos endometriais, estas perdem significância quando são realizados estudos de regressão logística multivariada ajustada para a idade ${ }^{6,9}$. No presente estudo, as pacientes com pólipo endometrial apresentaram idade mais avançada, com maior idade da menopausa e tempo de menopausa quando comparadas ao controle. Diversos estudos demonstraram risco maior para o desenvolvimento de pólipos e do câncer de endométrio com o avançar da idade e com o tempo transcorrido de menopausa, sendo tais fatores considerados como fatores de risco independentes para o desenvolvimento dessas condições ${ }^{7,9}$.

Neste estudo, observou-se que uma maior porcentagem de mulheres com pólipo era obesa $(72 \%)$ quando comparadas as mulheres sem alterações endometriais (39\%). Concomitantemente, a medida da cintura também foi superior entre as pacientes com pólipo endometrial. A obesidade androgênica ou central é metabolicamente diferente da ginecoide, pois associa-se às maiores taxas de lipólise tendo repercussões negativas sobre o perfil lipídico, com aumento dos ácidos graxos livres e TG e redução dos valores de $\mathrm{HDL}^{26-28}$. Os ácidos graxos livres, ocupando os receptores de insulina, induzem a RI por interferirem com o sinal de transcrição e, portanto, no transporte de glicose $^{22}$. A insulina em altas concentrações liga-se aos receptores do fator de crescimento semelhante à insulina (IGF-I), elevando à produção de androgênios, a qual, somada à redução da proteína carreadora dos hormônios sexuais (SHBG), ocasionada pela obesidade, causa aumento na biodisponibilidade dos estrogênios, principalmente da estrona ${ }^{29}$. No endométrio, o incremento do IGF-I representa também um possível fator de proliferação local, acarretando em alterações endometriais ${ }^{30}$.

Pinheiro et al. ${ }^{24}$ avaliaram, com métodos de imunoistoquímica, a expressão dos receptores de estrogênio (RE), dos receptores de progesterona (RP), Bcl-2, ciclo-oxigenase-2 (COX-2) e Ki67 em pólipos endometriais e sua associação com a obesidade. Foram incluídas 515 mulheres na pré e pós-menopausa submetidas à histeroscopia com diagnóstico histológico de pólipos benignos. Entre aquelas na pós-menopausa, a imunoexpressão da COX-2 e Bcl-2 no epitélio glandular foi mais frequente em obesas do que em não obesas. Pólipos de obesas tiveram expressão mais intensa do RP nos compartimentos glandulares e estromais. Tais dados sugerem que a etiologia e patogênese de pólipos em mulheres obesas parecem estar associadas com o RP, a inibição de apoptose e os mecanismos celulares associados com a inflamação ${ }^{24}$. Em estudo realizado por Gao et al. ${ }^{31}$, concentrações mais elevadas de leptina, causadas pela obesidade, estão relacionadas com o aumento da produção e ação da COX-2, que, por sua vez, leva ao aumento da proliferação celular. Esse mecanismo pode explicar a maior frequência de pólipos endometriais em mulheres obesas ${ }^{31}$.

$\mathrm{Na}$ presente pesquisa, observou-se incidência mais elevada de diabetes, hipertensão e dislipidemias $(28,8$; 75,8 e $56,1 \%$; respectivamente) entre as pacientes com pólipo endometrial quando comparadas ao controle (6,8; 40,9 e $13,3 \%$; respectivamente). Uma menor porcentagem de mulheres com pólipo endometrial apresentava valores considerados ótimos de HDL e glicose quando comparadas ao controle $(47,7$ versus $62,5 \%$ e 47,7 versus $79,2 \%$; respectivamente). RI e distúrbios do metabolismo de carboidratos são comuns em mulheres com doenças endometriais ${ }^{12}$. Dos componentes envolvidos na SM, a obesidade abdominal pode ser considerada como o elemento-chave no desenvolvimento da neoplasia endometrial, porém parece haver também significância nessa associação com hiperglicemia, hipertrigliceridemia e hipertensão $\operatorname{arterial}^{15}$.

As mulheres com pólipo endometrial tiveram mais filhos quando comparadas ao Grupo Controle (média de 3,8 versus 2,8 filhos). Além disso, a análise de risco demonstrou maior chance de desenvolvimento do pólipo endometrial em pacientes que tinham pelo menos um filho. A multiparidade é classicamente considerada como o fator protetor para doenças endometriais, ao diminuir o estímulo estrogênico no endométrio durante a gestação ${ }^{32}$. No entanto, o achado neste estudo de que a multiparidade estaria associada a um risco aumentado para o desenvolvimento do pólipo de endométrio deve ser analisado com cautela, uma vez que a maioria da população estudada foi constituída por mulheres multíparas em ambos os grupos (66,7 versus 54,6\%). Essa característica epidemiológica da população local pode ser considerada como um viés deste resultado.

O uso de TH em mulheres na pós-menopausa parece reduzir o desenvolvimento dos pólipos endometriais, ao causar diminuição da atividade proliferativa e estimular 
a apoptose ${ }^{33-35}$. Esse efeito é melhor observado quando utilizam-se progestagênios com alta ação antiestrogênica $^{34}$. O uso de TH em mulheres na pós-menopausa portadoras de SM tem efeito positivo sobre a RI e reduz o acúmulo de gordura visceral ${ }^{17,36}$. Em mulheres com $\mathrm{SM}$, os benefícios observados são decorrentes, principalmente, da ação estrogênica, sendo indicado o uso de progestagênios neutros para o melhor resultado da $\mathrm{TH}^{37}$. Neste estudo, no entanto, não foi observada nenhuma relação no desenvolvimento do pólipo endometrial com o uso de TH. Ressalta-se que a pequena porcentagem das usuárias de TH em ambos os grupos (16,7 nos casos versus $22 \%$ nos controles) pode ter sido um fator limitante nessa observação.

As alterações hormonais e inflamatórias da SM parecem estar intrinsicamente correlacionadas com as condições predisponentes para o desenvolvimento de doenças do endométrio. O avançar da idade e o estado pós-menopáusico podem ser considerados como gatilhos para uma série de modificações micromoleculares que irão conduzir a distúrbios biorreguladores do organismo feminino durante o envelhecimento. Uma compreensão dessa microesfera em futuras pesquisas poderá aprimorar o tratamento e o acompanhamento, hoje preconizados, das mulheres na pós-menopausa, sobretudo naquelas que apresentam alterações endometriais. Apesar da contínua controvérsia na literatura, este estudo demonstrou que, em mulheres na pós-menopausa, obesidade, dislipidemia, hiperglicemia e presença de SM foram fatores preditivos para o desenvolvimento de pólipo endometrial.

\section{Agradecimentos}

Ao Grupo de Apoio à Pesquisa (GAP) da Faculdade de Medicina de Botucatu da Universidade Estadual Paulista "Júlio de Mesquita Filho”, em especial a José Eduardo Corrente, pela assessoria estatística e análise dos resultados do estudo.

\section{Referências}

1. Anastasiadis PG, Koutlaki NG, Skaphida PG, Galazios GC, Tsikouras PN, Liberis VA. Endometrial polyps: prevalence, detection, and malignant potential in women with abnormal uterine bleeding. Eur J Gynaecol Oncol. 2000;21 (2): 180-3.

2. Dreisler E, Stampe Sorensen S, Ibsen PH, Lose G. Prevalence of endometrial polyps and abnormal uterine bleeding in a Danish population aged 20-74 years. Ultrasound Obstet Gynecol. 2009;33(1):102-8.

3. Haimov-Kochman R, Deri-Hasid R, Hamani Y, Voss E. The natural course of endometrial polyps: could they vanish when left untreated? Fertil Steril. 2009;92(2):828.e 1 1-2.

4. Wethington SL, Herzog TJ, Burke WM, Sun X, Lerner JP, Lewin SN, et al. Risk and predictors of malignancy in women with endometrial polyps. Ann Surg Oncol. 2011 ; 18(13):3819-23.

5. Golan A, Sagiv R, Berar M, Ginath S, Glezerman M. Bipolar electrical energy in physiologic solution-a revolution in operative hysteroscopy. J Am Assoc Gynecol Laparosc. 2001;8(2):252-8.

6. Salim S, Won H, Nesbitt-Hawes E, Campbell N, Abbott J. Diagnosis and management of endometrial polyps: a critical review of the literature. J Minim Invasive Gynecol. $2011 ; 18(5): 569-81$.

7. Dias DS, Bueloni-Dias FN, Dias R, Nahás-Neto J, Petri Nahás EA, Leite NJ, et al. Usefulness of clinical, ultrasonographic, hysteroscopic, and immunohistochemical parameters in differentiating endometrial polyps from endometrial cancer. J Minim Invasive Gynecol. 2014;21(2):296-302

8. Cohen I. Endometrial pathologies associated with postmenopausal tamoxifen treatment. Gynecol Oncol. 2004;94(2):256-66.

9. Nappi L, Indraccolo U, Di Spiezio Sardo A, Gentile G, Palombino K, Castaldi MA, et al. Are diabetes, hypertension, and obesity independent risk factors for endometrial polyps? J Minim Invasive Gynecol. 2009;16(2):157-62.
10. Schneider JG, Tompkins C, Blumenthal RS, Mora S. The metabolic syndrome in women. Cardiol Rev. 2006;14(6):286-91.

11. Solymoss BC, Bourassa MG, Marcil M, Levesque S, Varga S, Campeau L. Long-term rates of cardiovascular events in patients with the metabolic syndrome according to severity of coronaryangiographic alterations. Coron Artery Dis. 2009;20(1):1-8.

12. Kacalska-Janssen $O$, Rajtar-Ciosek A, Zmaczyński A, Wyroba J, Milewicz T, Krzyczkowska-Sendrakowska M, et al. Markers of insulin resistance in perimenopausal women with endometrial pathology. Ginekol Pol. 2013;84(1 1):922-9.

13. Weiderpass E, Persson I, Adami HO, Magnusson C, Lindgren A, Baron JA. Body size in different periods of life, diabetes mellitus, hypertension, and risk of postmenopausal endometrial cancer (Sweden). Cancer Causes Control. 2000; 11 (2): 185-92.

14. Anderson KE, Anderson E, Mink PJ, Hong CP, Kushi LH, Sellers TA, et al. Diabetes and endometrial cancer in the lowa women's health study. Cancer Epidemiol Biomarkers Prev. 2001;10(6):611-6.

15. Esposito K, Chiodini P, Capuano A, Bellastella G, Maiorino MI, Giugliano D. Metabolic syndrome and endometrial cancer: a meta-analysis. Endocrine. 2014;45(1):28-36.

16. Miranda SMN, Gomes MT, Silva IDCG, Girão MJBC. [Endometrial polyps: clinical and epidemiological aspects and analysis of polymorphisms]. Rev Bras Ginecol Obstet. 2010;32(7):327-33. Portuguese.

17. Expert Panel on Detection, Evaluation and Treatment of High Blood Cholesterol in Adults. Executive summary of the third report of The National Cholesterol Education Program (NCEP) expert panel on detection, evaluation, and treatment of high blood cholesterol in adults (Adult Treatment Panel III). JAMA. 2001 ;285(19):2486-97.

18. Friedewald WT, Levy RI, Fredrickson DS. Estimation of the concentration of low-density lipoprotein cholesterol in plasma, without use of the preparative ultracentrifuge. Clin Chem. 1972;18(6):499-502. 
19. Ridker PM. Cardiology patient page. C-reactive protein: a simple test to help predict risk of heart attack and stroke. Circulation. 2003; 108(12):e81-5.

20. Hartman A, Wolfman W, Nayot D, Hartman M. Endometrial thickness in 1,500 asymptomatic postmenopausal women not on hormone replacement therapy. Gynecol Obstet Invest. 2013;75(3):191-5.

21. Dias DS, Bueloni-Dias FN, Dias R, Nahás-Neto J, Nahás EAP. Pólipos endometriais e seu risco de malignização: aspectos epidemiológicos, clínicos e imunoistoquímicos. Femina. 2013;41(1):33-8.

22. Dandona P, Aljada A, Chaudhuri A, Mohanty P, Garg R. Metabolic syndrome: a comprehensive perspective based on interactions between obesity, diabetes, and inflammation. Circulation. 2005; 111 (11):1448-54.

23. Nahas EAP, Almeida BR, Buttros DAB, Véspoli HDL, Uemura G, Nahas-Neto J. [Metabolic syndrome in postmenopausal breast cancer survivors]. Rev Bras Ginecol Obstet. 2012;34(12):555-62. Portuguese.

24. Pinheiro A, Antunes A Jr, Andrade L, De Brot L, Pinto-Neto AM, Costa-Paiva L. Expression of hormone receptors, Bcl2, Cox2 and $\mathrm{Ki} 67$ in benign endometrial polyps and their association with obesity. Mol Med Rep. 2014;9(6):2335-41.

25. Figueiredo Neto JA, Figuerêdo ED, Barbosa JB, Barbosa FF, Costa GRC, Nina VIS, et al. Síndrome metabólica e menopausa: estudo transversal em ambulatório de ginecologia. Arq Bras Cardiol. 2010;95(3):339-45.

26. Berg G, Mesch V, Boero L, Sayegh F, Prada M, Royer M, et al. Lipid and lipoprotein profile in menopausal transition. Effects of hormones, age and fat distribution. Horm Metab Res. 2004;36(4):215-20.

27. Grundy SM, Brewer HB Jr, Cleeman Jl, Smith SC Jr, Lenfant C; American Heart Association; National Heart, Lung, and Blood Institute. Definition of metabolic syndrome: report of the National Heart, Lung, and Blood Institute/American Heart Association conference on scientific issues related to definition. Circulation. 2004; 109(3):433-8.
28. Zhang C, Rexrode KM, van Dam RM, Li TY, Hu FB. Abdominal obesity and the risk of all-cause, cardiovascular, and cancer mortality: sixteen years of follow-up in US women. Circulation. 2008; 117(13): 1658-67.

29. Webb PM. Commentary: weight gain, weight loss, and endometrial cancer. Int J Epidemiol. 2006;35(1):166-8.

30. Serin IS, Ozçelik B, Basbug M, Ozsahin O, Yilmazsoy A, Erez R. Effects of hypertension and obesity on endometrial thickness. Eur J Obstet Gynecol Reprod Biol. 2003;109(1):72-5.

31. Gao J, Tian J, Lv Y, Shi F, Kong F, Shi H, et al. Leptin induces functional activation of cyclooxygenase-2 through JAK2/STAT3, MAPK/ERK, and PI3K/AKT pathways in human endometrial cancer cells. Cancer Sci. 2009; 100(3):389-95.

32. Bakour SH, Gupta JK, Khan KS. Risk factors associated with endometrial polyps in abnormal uterine bleeding. Int J Gynaecol Obstet. 2002;76(2): 165-8.

33. Maia H Jr, Maltez A, Studard E, Athayde C, Coutinho EM. Effect of previous hormone replacement therapy on endometrial polyps during menopause. Gynecol Endocrinol. 2004;18(6):299-304.

34. Oguz S, Sargin A, Kelekci S, Aytan H, Tapisiz OL, Mollamahmutoglu L. The role of hormone replacement therapy in endometrial polyp formation. Maturitas. 2005;50(3):231-6.

35. Gardner FJ, Konje JC, Bell SC, Abrams KR, Brown L, Taylor DJ, et al. Prevention of tamoxifen induced endometrial polyps using a levonorgestrel releasing intrauterine system longterm follow-up of a randomised control trial. Gynecol Oncol. $2009 ; 114(3): 452-6$.

36. Stevenson JC. Type and route of estrogen administration. Climacteric. 2009; 12(Suppl 1):86-90.

37. Meirelles RMR. [Menopause and metabolic syndrome]. Arq Bras Endocrinol Metab. 2014;58(2):91-6. Portuguese. 